\title{
Aplicación de un programa de estrategias para la comprensión lectora
}

\section{Application of a program for reading comprehension strategies}

\section{RESUMEN}

Objetivos: Aplicar un programa de estrategias para mejorar la comprensión lectora y el aprendizaje en estudiantes del primer grado del Colegio Particular "Ingeniería" de El Tambo Huancayo. Métodos: El diseño aplicado es cuasi experimental con pre y post prueba. El instrumento de medición es el test de comprensión lectora. La muestra estuvo conformada por 40 estudiantes del primer grado del Colegio Privado "Ingeniería"; de los cuales, 20 de ellos, integran al grupo experimental y 20, al grupo control. En el primer grupo, se aplicó un programa de estrategias para mejorar la comprensión lectora y el aprendizaje; en el segundo grupo, no se aplicó dicho programa. Resultados: Se demostró que el programa de estrategias mejoró significativamente la comprensión lectora y el aprendizaje, al encontrar que el grupo experimental en la prueba de entrada obtuvo una media de 11,25 y en la prueba de salida una media de 19,5 diferencia significativa al 0,05 (tc $=17,6)$; mientras que el grupo control en la prueba de entrada logró una media de 10,1 y en la prueba de salida una media de 10,7. Conclusiones: La aplicación de un programa de estrategias metodológicas ajustadas a una debida planificación da resultados positivos para la comprensión lectora y el aprendizaje, con un nivel de $95 \%$ de confiabilidad y margen de error de $0,05 \%$ en los estudiantes del Primer Grado de Educación Secundaria del Colegio Particular "Ingeniería" de El Tambo, Huancayo.

Palabras clave: Programa, estrategias cognitivas, comprensión lectora.

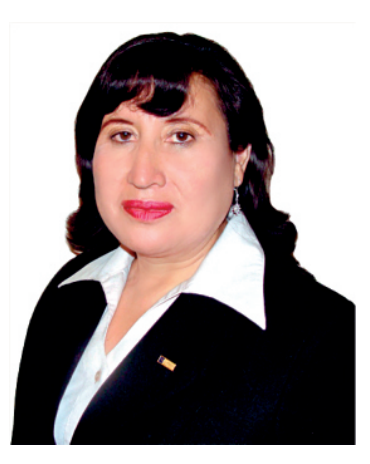

Virginia Delia Navarro Salvador Universidad Continental de Ciencias e Ingeniería

\section{ABSTRACT}

Objective: To apply a Strategies Program to improve the reading comprehension and learning in students of first grade from "Ingeniería" private high school from El Tambo Huancayo. Method: The design of this investigation was quasi- experimental with pre and post test. The measuring instrument was the test of reading comprehension. The sample was conformed by 40 students of first grade of "Ingeniería" private high school; where 20 of them were the control group, and 20 students were the experimental group. In the first group, the strategies program was implemented in order to improve reading comprehension and learning, in the second group, this program was not applied. Results: It was

1 Mg. en Ciencias de la Educación, especialidad de Problemas de Aprendizaje. Docente de la Universidad Continental de Ciencias e Ingeniería. 
demonstrated that the strategies program improved significantly the reading understanding and learning, when was an average of 11,25 and in the final test an average of 19,5 significant difference to the 0,05 (tc $=17,6$ ) while the control group in the entrance test obtained an average of 10,1 and in the final test obtained an average of 10,7. Conclusions: The implementation of a program in a methodological strategies adjusted to a proper planning gives positive results for reading comprehension and learning, with 95\% of confidence level and margin of error of $0,05 \%$ in the students of first grade of secondary education in "Ingeniería" private high school from El Tambo, Huancayo.

Key words: Program, cognitive strategies, reading comprehension.

\section{INTRODUCCIÓN}

Siendo la lectura la base para la enseñanza aprendizaje general y habiéndose diagnosticado un nivel bajo de comprensión de lectura en los estudiantes del primer grado del Colegio Ingeniería, es de mi preocupación realizar el presente trabajo de investigación para contribuir mediante aportes principales de técnicas, estrategias de lectura y formar buenos lectores.

Conociendo la gran importancia y la trascendencia que tiene la lectura en el desarrollo de todas las demás habilidades del educando. Vallés, considera, leer es un esfuerzo en busca de significado, es una construcción activa del sujeto mediante el uso de todo tipo de claves y estrategias (1); por lo tanto, en este trabajo de investigación se aplica un programa de estrategias para la comprensión lectora y el aprendizaje en estudiantes del primer grado de educación secundaria del Colegio Particular "Ingeniería" de El Tambo, Huancayo.

La investigación realizada en "Velocidad y comprensión lectora" se intenta dar solución a un problema grave que ha existido en la población escolar de nivel secundario; la deficiencia lectora que comprende una lectura lenta y un nivel bajo de comprensión. El objetivo general que se plantea en la investigación es determinar el método que mejore la comprensión lectora desarrollando al mismo tiempo la velocidad para leer, logrando resultados positivos (2).
"Influencia de la enseñanza directa en el mejoramiento de la comprensión lectora en los estudiantes de la Facultad de Ciencias de la Educación de la Universidad Nacional San Cristóbal de Huamanga (UNSCH) (Ayacucho, Perú), plantea el problema de: si existen o no diferencias significativas en la comprensión lectora del grupo de estudiantes de la Escuela de Formación Profesional de Educación Inicial de la Facultad de Ciencias de la Educación. En conclusión, la estrategia enseñanza directa ha mejorado significativamente la comprensión lectora de los estudiantes del primer ciclo de la Escuela de Formación Profesional de Educación Inicial de la Facultad de Ciencias de la Educación de la UNSCH (3).

"Relación entre los niveles de comprensión lectora y el conocimiento de los participantes de un programa de formación docente a distancia de la Universidad Nacional José Faustino Sánchez Carrión de Huacho. En la investigación se utilizó el Test CLOZE para determinar los niveles de comprensión lectora. Los resultados y conclusiones principales establecen que existe una asociación entre los puntajes de comprensión lectora y las notas de rendimiento general de los estudiantes" (4)

"Relaciones entre la inteligencia general, el rendimiento académico y la comprensión de lectura en el campo educativo" plantea la interrogante sobre el grado de correlación entre los puntajes obtenidos en una muestra de estudiantes de Educación Secundaria y de la Universidad, aplicó un test de inteligencia general en una prueba de comprensión lectora y rendimiento académico, utilizó una prueba de comprensión lectora tipo SAT (Scholastic Aptitude Test). Las conclusiones en la inteligencia general y la comprensión lectora presentan una correlación medianamente alta y significativa en el campo educacional (5)

El problema de investigación es: ¿̇De qué manera la aplicación de un programa de estrategias mejora la comprensión lectora y el aprendizaje en los estudiantes del primer grado de educación secundaria del Colegio Particular "Ingeniería" de El Tambo, Huancayo?

La hipótesis de investigación es: La aplicación adecuada del programa de estrategias mejorará la comprensión lectora y el aprendizaje en estudiantes del primer grado de educación secundaria del Colegio Particular "Ingeniería" de 
El Tambo, Huancayo. El objetivo es: Aplicar un programa de estrategias para el mejoramiento de la comprensión lectora y el aprendizaje en estudiantes del primer grado de educación secundaria del Colegio Particular "Ingeniería" de El Tambo Huancayo.

La comprensión de textos es una actividad compleja de carácter estratégico, por lo tanto, este trabajo tiene como fin contribuir en el desarrollo de habilidades en la lectura y demostrar la eficacia de la aplicación de un programa de estrategias para mejorar la comprensión lectora y el aprendizaje en los estudiantes del primer grado de educación secundaria del Colegio Particular "Ingeniería" de El Tambo, Huancayo.

\section{MATERIAL Y MÉTODOS}

Habilidades y número de ítems que se evalúan en el test de Violeta Tapia:

\begin{tabular}{|l|l|}
\hline \multicolumn{1}{|c|}{ Habilidades } & \multicolumn{1}{c|}{ items } \\
\hline $\begin{array}{l}\text { Información de hechos } \\
\text { Definición de significado }\end{array}$ & $1,2,13,24,27,29$ \\
\hline $\begin{array}{l}\text { Identificación de la } \\
\text { idea central del texto }\end{array}$ & $14,23,28$ \\
\hline & $4,6,7,9,10,12$, \\
\hline Interpretación de hechos & $15,17,18$, \\
\hline Inferencia sobre el autor & $21,22,31,32,36$ \\
\hline $\begin{array}{l}\text { Inferencia sobre el } \\
\text { contenido del fragmento }\end{array}$ & 37,38 \\
\hline Rotular & $19,20,25,30,34$ y 35 \\
\hline
\end{tabular}

En el grupo experimental se ha aplicado un programa de estrategias sencillas para mejorar la comprensión lectora y el aprendizaje adecuado.

El programa planteado tiene por finalidad que el lector, relacione de manera organizada la información enlazando lo que conoce con lo que va a conocer, teniendo como punto de encuentro el aprendizaje y el recuerdo; es decir, el énfasis y la evocación.

Se intenta desarrollar en los estudiantes el hábito de aplicar estrategias de comprensión lectora relacionándolos con textos que contengan temas similares o de contexto. Los módulos aplicados son:

Módulo 1: "La educación", módulo 2: "Los Chinchilpos y los Gamonales", módulo 3: "Maruja", módulo 4: "El río Mantaro", módulo 5: "La Psicología de la educación", módulo 6: "La Psicología de la educación", módulo 7: "Amor, amor", módulo 8: "Ñawinpuquio", módulo 9: "El niño indefenso", módulo 10: "El dengue", módulo 11: "Comentarios Reales de los Incas", y el módulo 12: "Perú pueblo de indios".

Los objetivos del programa de comprensión lectora son: a) Adquirir conocimientos básicos sobre el proceso de comprensión lectora, la importancia en la lectura y el aprendizaje, y conocimientos meta cognitivos sobre la comprensión lectora y el aprendizaje, b) Asimilar el aprendizaje de diferentes estrategias de comprensión lectora: el modo y la oportunidad de su aplicación.

Para conseguir estos objetivos se han diseñado una serie de actividades que los estudiantes han realizado con distintos tipos de textos, en cuanto a contenido y grado de dificultad. Los componentes del programa son: Conocimientos sobre comprensión lectora y procesos psicológicos implicados, recuerdo inmediato y atención sostenida, activación de conocimientos previos, conocimientos sobre las estructuras de los textos, selección de la información relevante del texto y meta comprensión. Debido a su finalidad y naturaleza la investigación que se desarrolló se encuentra dentro de la investigación de tipo aplicada.

En concordancia al tipo y nivel de investigación, se utilizó el método científico, específicamente el método experimental, en su variante: Diseño cuasi experimental de dos grupos no equivalentes. Mientras en el grupo control solo se aplicó el pre test, no se les aplicó el programa de estrategias para la comprensión lectora y el aprendizaje; por lo tanto, ellos desconocen dicho programa.

Al finalizar el año se les aplicó el mismo test de comprensión de lectura obteniendo resultados deficientes. 


\section{Población}

El estudio se desarrolló en los estudiantes del Colegio Particular "Ingeniería" del El Tambo, Huancayo, la población estuvo conformada por todos los estudiantes que fueron matriculados en el primer grado de secundaria con un total de 126 estudiantes, promedio de 12 años de edad; 70 de género masculino y 56 femenino.

\section{Muestra}

La muestra estuvo constituido por 40 estudiantes; 20 del grupo control y 20 del grupo experimental, matriculados en el primer grado del Colegio Particular "Ingeniería".

La muestra es no probabilística intencionada, no aleatoria; la técnica y procedimiento de selección es intencional porque responde a las necesidades e intereses del investigador, se ha conformado por igualación con el grupo experimental, de los cuales se tomó dos grupos: Un grupo experimental de 20 alumnos: 10 de género masculino y 10 femenino; y otro grupo de control de 20 alumnos: 13 de género masculino y 07 , femenino.

\begin{tabular}{ccccccc} 
Grupo & Masc. & $\%$ & Fem. & $\%$ & Total & $\%$ \\
\hline Experimental & 10 & 25 & 10 & 25 & 20 & 50 \\
Control & 13 & 33 & 07 & 17 & 20 & 50 \\
Total & & & & & 40 & 100 \\
\hline
\end{tabular}

\section{RESULTADOS}

Los resultados de la prueba de entrada nos indica que existe una deficiencia en la comprensión lectora y el aprendizaje en los estudiantes del grupo control y grupo experimental, lo que se demuestra a través de la $t_{c}<t_{t}$; es decir, que la $t_{t}$ es mayor que la $t_{c}$ obteniéndose un resultado negativo en la comprensión de lectura, así como el desconocimiento de estrategias para la comprensión; es decir 1,14<2,042.

En la tabla 1 se percibe el incremento de las puntuaciones en la media aritmética de 19,5 del grupo experimental con una desviación de 0,76 en donde los datos están más concentrados alrededor de la media aritmética del grupo control que es 10,7; demostrándose que el sesgo es positivo para el grupo experimental, demostrado a través de la $t_{c}>t_{t}$, es decir 17,6> 2,042 , notándose una significancia a un $95 \%$, con un margen de error al 0,05.

\section{Prueba de hipótesis con la "t" de student}

La " $t$ " de student es una prueba estadística para evaluar si dos grupos difieren entre si de manera significativa respecto a sus medias aritméticas. Para determinar la " $t$ " de student se considera los estadígrafos obtenidos en la prueba de salida de los grupos experimental y control. Hallando la $\dagger$ en la tabla con $38 \mathrm{GL}$ y un nivel de significación de 0,05 es 2,042 a un nivel de confianza de 95\%. El valor calculado de la tc $=17,6$ resultando superior al valor de la tabla $t t=2,042$. Es decir: tc $>\mathrm{tt} ; 17,6>2,042$.

Tabla 1. Resultados de la prueba de entrada y salida de los alumnos del grupo control y experimental.

\begin{tabular}{|c|c|c|c|c|}
\hline \multirow[b]{2}{*}{ Grupos de estudio } & \multicolumn{2}{|c|}{ PRE TEST } & \multicolumn{2}{|c|}{ POST TEST } \\
\hline & $x$ & DS & $x$ & DS \\
\hline Grupo experimental & 11,25 & 2,51 & 19,50 & 0,76 \\
\hline \multirow[t]{2}{*}{ Grupo control } & 10,10 & 3,58 & 10,70 & 2,05 \\
\hline & \multicolumn{2}{|c|}{1,14} & \multicolumn{2}{|c|}{17,6} \\
\hline
\end{tabular}


En conclusión existe significancia en la aplicación de un programa de estrategias para mejorar la comprensión lectora y el aprendizaje.

\section{DISCUSIÓN}

Los resultados de la prueba de entrada, nos muestran que algunos estudiantes del primer grado del Colegio Particular "Ingeniería" de El Tambo tienen deficiencias en la comprensión lectora y el aprendizaje.

El programa de comprensión lectora, orienta a los estudiantes a desarrollar estrategias de comprensión lectora relacionándolos con textos que contengan temas similares o de contexto. Los resultados observados nos indican que los items referidos a la comprensión lectora y el aprendizaje, alcanzaron coeficientes con resultados significativos, así como valores de confiabilidad adecuados, estos resultados concuerdan con Bañuelos (2) "Velocidad y comprensión lectora", que concluye que la aplicación del método de lectura veloz mejora la comprensión lectora en los alumnos de secundaria.

En el ámbito nacional la investigación realizada coincide con Cabanillas (3) "Influencia de la enseñanza directa en el mejoramiento de la comprensión lectora de los estudiantes de la Facultad de Ciencias de la Educación de la UNSCH (Ayacucho), grupo que trabaja con la estrategia didáctica enseñanza directa, con respecto al grupo de estudiantes al cual no se le aplica dicha estrategia. Concluye que la estrategia enseñanza directa ha mejorado significativamente la comprensión lectora de los estudiantes del primer ciclo de la Escuela de Formación Profesional de Educación Inicial de la Facultad de Ciencias de la Educación de la UNSCH.

Aliaga (4) "Relación entre los niveles de comprensión lectora y el conocimiento de los participantes de un Programa de Formación Docente a distancia". Las conclusiones principales establecen que existe una asociación entre los puntajes de comprensión lectora y las notas de rendimiento general de los estudiantes. Asimismo, que los textos son interpretados de acuerdo al esquema mental de cada estudiante, observándose que los resultados son mejores cuando el contenido es parte de su experiencia. Es decir, en su gran mayoría no poseen buena comprensión lectora, hecho que es muy grave tratándose de formadores de futuras generaciones.

Miljanovich (5) "Relaciones entre la inteligencia general, el rendimiento académico y la comprensión de lectura en el campo educativo". La conclusión más importante en relación a nuestra propia investigación es que la inteligencia general y la comprensión lectora presentan una correlación medianamente alta y significativa en el campo educacional.

En dichas investigaciones y comparaciones podemos deducir que en los trabajos de investigación mencionados se demuestra que el empleo de las técnicas citadas han mejorado la comprensión lectora. Asimismo, en el trabajo de investigación realizado, el programa de estrategias ha permitido relacionar los procesos de la comprensión lectora; es decir, ya no de manera espontánea, sino considerando cada una de las habilidades que se requiere. En síntesis los hallazgos observados justifican la necesidad de fomentar la aplicación del programa de estrategias para mejorar la comprensión lectora y el aprendizaje en los estudiantes del primer grado del Colegio Particular "Ingeniería" de El Tambo - Huancayo. 


\section{REFERENCIAS BIBLIOGRAFCAS}

1. Vallés A. Técnicas de velocidad y comprensión Lectora. Madrid: Editorial Escuela Española. S.A.; 1995.

2. Bañuelos M. Velocidad y comprensión lectora. [Tesis para optar el grado de Maestría en metodología de la enseñanza]. Zacatecas: Universidad de Valparaíso; 2003.

3. Cabanillas G. Influencia de la enseñanza directa en el mejoramiento de la comprensión lectora en los estudiantes de la facultad de Ciencias de la Educación de la UNSCH. [Tesis para optar el grado de Doctor]. Lima: Universidad Mayor de San Marcos. Facultad de Educación. Escuela de Post-grado; 2004.

4. Aliaga N. Relación entre los niveles de comprensión lectora y el conocimiento de los participantes de un programa de formación docente a distancia (Tesis para optar el Grado de Maestría). Lima: Universidad Nacional José Faustino Sánchez Carrión. Huacho; 2000.

5. Miljanovich M. Relaciones entre la inteligencia general, el rendimiento Académico y la comprensión lectora en el campo educativo [Tesis para optar el grado de Doctor en Educación] Lima: Universidad Nacional Mayor San Marcos; 2000.

Correo electrónico:

vnavarro@continental.edu.pe 